\title{
Impact of NSGA-II Objectives on EEG Feature Selection Related to Motor Imagery
}

\author{
Miguel Leon \\ School of Innovation, Design and \\ Technology \\ Vasteras, Sweden \\ miguel.leonortiz@mdh.se
}

\author{
Christoffer Parkkila \\ School of Innovation, Design and \\ Technology \\ Vasteras, Sweden \\ cpa16002@student.mdh.se
}

\author{
Jonatan Tidare \\ School of Innovation, Design and \\ Technology \\ Vasteras, Sweden \\ jonatan.tidare@mdh.se
}

\author{
Ning Xiong \\ School of Innovation, Design and \\ Technology \\ Vasteras, Sweden \\ ning.xiong@mdh.se
}

\author{
Elaine Astrand \\ School of Innovation, Design and \\ Technology \\ Vasteras, Sweden \\ Elaine.Astrand@mdh.se
}

\begin{abstract}
The selection of ElectroEncephaloGram (EEG) features with functional relevance to Motor Imagery (MI) is a crucial task for successful outcome in Brain-Computer Interface (BCI)-based motor rehabilitation. Individual EEG patterns during $\mathrm{MI}$ requires subjectdependent feature selection, which is an arduous task due to the complexity and large number of features. One solution is to use metaheuristics, e.g. Genetic Algorithm (GA), to avoid an exhaustive search which is impractical. In this work, one of the most widely used GA, NSGA-II, is used with an hierarchical individual representation to facilitate the exclusion of EEG channels irrelevant for MI. In essence, the performance of different objectives in NSGA-II was evaluated on a previously recorded MI EEG data set. Empirical results show that k-Nearest Neighbors (k-NN) combined with Pearson's Correlation (PCFS) as objective functions yielded higher classification accuracy as compared to the other objectivecombinations (73\% vs. 69\%). Linear Discriminant Analysis (LDA) combined with Feature Reduction (FR) as objective functions maximized the reduction of features $(99.6 \%)$ but reduced classification performance $(65.6 \%)$. All classifier objectives combined with PCFS selected similar features in accordance with expected activity patterns during MI. In conclusion, PCFS and a classifier as objective functions constitutes a good trade-off solution for MI data.
\end{abstract}

\section{CCS CONCEPTS}

- Computing methodologies $\rightarrow$ Feature selection; • Applied computing $\rightarrow$ Life and medical sciences;

Permission to make digital or hard copies of all or part of this work for personal or classroom use is granted without fee provided that copies are not made or distributed for profit or commercial advantage and that copies bear this notice and the full citation on the first page. Copyrights for components of this work owned by others than ACM must be honored. Abstracting with credit is permitted. To copy otherwise, or republish, to post on servers or to redistribute to lists, requires prior specific permission and/or a fee. Request permissions from permissions@acm.org.

GECCO '20, July 8-12, 2020, Cancún, Mexico

(C) 2020 Association for Computing Machinery.

ACM ISBN 978-1-4503-7128-5/20/07 ..\$15.00

https://doi.org/10.1145/3377930.3390198

\section{KEYWORDS}

ElectroEncephaloGram (EEG), Mental Imagery, Feature Selection, Fast Elitist Non-Dominated Sorting Genetic Algorithm (NSGA-II)

\section{ACM Reference Format:}

Miguel Leon, Christoffer Parkkila, Jonatan Tidare, Ning Xiong, and Elaine Astrand. 2020. Impact of NSGA-II Objectives on EEG Feature Selection Related to Motor Imagery. In Genetic and Evolutionary Computation Conference (GECCO '20), July 8-12, 2020, Cancún, Mexico. ACM, New York, NY, USA, 9 pages. https://doi.org/10.1145/3377930.3390198

\section{INTRODUCTION}

ElectroEncephaloGraphy (EEG) has many advantages and is often used to study and extract temporally dynamic patterns in the brain. Specifically, frequency transformation of EEG signals has shown to reveal underlying functional mechanisms [27] with the downside of producing a large number of features. Selecting features from EEG signals relevant to a specific function is therefore important in EEG research.

In all types of EEG Brain-Computer Interface (BCI) research, ranging from control of an external device to rehabilitation using real-time neurofeedback, it is essential to rapidly and accurately extract information. One of the most widely used approaches to $\mathrm{BCI}$ applications is Motor Imagery (MI) which has shown promise in motor rehabilitation after stroke [29]. The technique is based on findings that brain regions involved in the imagination of motor actions largely overlap to those active when the motor action itself is performed [11]. When performing MI, a decrease in EEG oscillatory power, referred to as event-related desynchronization (ERD), over the sensorimotor area, in the $\mathrm{mu}(8-12 \mathrm{~Hz})$ and central beta (14-25 $\mathrm{Hz}$ ) rhythms is observed [28]. However, the exact ERD frequency range, spatial location and time course varies between individuals [34] which suggests that individual selection of the most relevant features is necessary for optimal extraction.

The selection is often performed by first considering a large number of features and then applying some method to select the most relevant ones. As with all high-dimensional data sets such as EEG rhythms, searching for an optimal solution (feature subset) by the use of grid-search is exhaustive and not feasible for real-time BCI applications. Thus, the use of metaheuristics has been developed to 
overcome this issue [3]. There are two main groups of metaheuristics: trajectory-based approaches (e.g. simulated annealing [21], iterative local search [32], hill climbing [33]) and population-based approaches (e.g. evolutionary algorithms [2], swarm intelligence [5]). Computationally speaking, trajectory-based algorithms are less expensive than population-based algorithms, however they tend to get stuck into local optima. On the contrary, populationbased approaches deal better with the stagnation problem, which is a frequently encountered in high dimensional search spaces. One of the most successful population-based approaches is Genetic Algorithms (GA)[13], which have shown the advantageous capability to efficiently explore the search space to find optimal or near-optimal solutions in many different applications $[14,25]$. One of the earliest use of GA for automated selection of EEG features has demonstrated the potentials of this technique in BCI applications [35]. Since then, the GA technique has been further developed to solve multi-objective optimization problems, involving more than one evaluation criteria (e.g. [37]). One way to solve the multi-objective problem is to weigh the objectives into a single-objective formulation [24]. However, the single-objective approach requires the practitioner to explicitly determine the trade-off between the objectives and does not offer any possibilities for exploring one objective at the expense of the other.

Instead, several different Multi-Objective Evolutionary Algorithms (MOEA) have been developed [26] that find multiple paretooptimal solutions, representing the best trade-offs between the objectives. Among them, the fast Elitist Non-Dominated Sorting Genetic Algorithm for Multi Objective Optimization (NSGA-II) is one of the most popular implementations of the MOEA concept [9]. In EEG research, the NSGA-II has demonstrated the ability to significantly reduce the number of features while retaining a high classification accuracy [15]. Specifically, the NSGA-II has been applied to increase the discriminability between EEG data recorded during different MI tasks (e.g. imagination of right versus left hand movements) while reducing the number of features (or channels) (e.g. [15]). In the majority of these studies, the classification accuracy or similar measure (e.g. kappa index) is reported to evaluate the feature selection. Only in the study by [19] the EEG channels selected by NSGA-II were examined and represented as topographical maps to show that electrodes over the sensorimotor area were selected, which in consideration to the MI tasks performed by the subjects were supported by previous EEG MI research [28]. When using a $\mathrm{BCI}$ for rehabilitation purposes, the functional role of the selected features will have fundamental impact on the outcome of the intervention and it is therefore crucial to examine the selected features when using an automated feature selection method.

In this work, a NSGA-II, using a hierarchical individual representation, proposed in [23] is adapted to investigate the impact of different objective sets for NSGA-II on the selection of features during a MI task. The classification accuracy and reduction of features are additionally used to evaluate the different objectives as well as a set of classifiers. For this purpose, the following experiments have been performed: (a) evaluation of the performance obtained by NSGA-II using different classifiers (k-NN [7], ANN [18], LDA [38] and SVM [33]), and using different combinations of objectives, (b) correlation analysis between different NSGA-II objectives and classifiers, and (c) similarity analysis of selected features by the different objective-combinations and their relevance to motor imagery.

The paper is structured as follows. First, a description of NSGA-II with a hierarchical representation is provided. Then, the experimental settings and implementation are described. Subsequently, the results of our empirical evaluation are presented followed by concluding remarks.

\section{NSGA-II WITH HIERARCHICAL INDIVIDUAL REPRESENTATION}

NSGA-II $[8,9]$ is a population-based algorithm, in which, each individual, $p_{i}$, of the population $P=\left\{p_{1}, p_{2}, \ldots, p_{i}, \ldots, p_{n}\right\}$, is a solution to the problem.

The initial step in NSGA-II is to create an initial population with random solutions to the specific problem to obtain the objectives' score. Then, a loop, called generation $(g)$, with 3 main operations follows.

- Firstly, $n$ different offsprings are generated through 3 breeding operations: parent selection, crossover, mutation.

- Secondly, the individuals from the population and the offsprings are divided into different groups, also called Pareto fronts, depending on if they are dominated by another individual or not. An individual, $i$ is considered to dominate another individual, $j$ if its score is higher at least for one objective and it is not worse in the rest of the objectives. Individuals inside the first front are not dominated by any other individual and those in the second front are only dominated by the individuals of the first front. This sorting operation is called Non-Dominated Sorting (NDS).

- The final operation is to assign the Crowding Distance (CD) to the different individuals of the same front. Higher score is assigned to solutions with longer distance to neighbouring solutions. The new population is composed with individuals from the first fronts. If a new front is added and the population size limit is surpassed, then, the solutions from the front, that did not fit, with less CD score will be removed until the population size limit is reached.

More information about CD assignment and NDS can be found in [9].

\subsection{Individual representation}

The intuitive way of representing a solution in GAs for EEG feature selection is to have one single vector in which each position of the vector represents a feature, thereby ignoring the spatial organization of the features. The value of each position is either 0 , if that feature is not selected, or 1 , if the feature is selected. This representation is suboptimal for removing one EEG channel completely since all features originating from that channel needs to be set to zero. For this reason, the probability of not selecting one complete channel is low.

In order to overcome this issue, the hierarchical individual representation, presented by [23], is used. This representation will have two related parts. First, the upper level is represented by a vector $\vec{X}=\left\{x_{1}, x_{2}, \ldots, x_{i}, \ldots, x_{c}\right\}$, where $x_{i} \in\{0,1\}$ and $c$ is the number of channels. In the lower level, each channel will have an associated 
vector $\vec{Y}_{i}=\left\{y_{i, 1}, y_{i, 2}, \ldots, y_{i, j}, \ldots, y_{i, f}\right\}$ where $y_{i, j} \in\{0,1\}$ and $f$ is the number of features. If $x_{i}$ is equal to 0 , then all the values of $Y_{i}$ will be set to zero, thereby completely removing one channel.

\section{$2.2 \quad$ Breeding operators}

As previously mentioned, $n$ different offsprings, $O=\left\{o_{1}, o_{2}, \ldots, o_{h}\right.$, $\left.\ldots, o_{n}\right\}$, are generated at each generation. In order to create the $h$-th offspring $\left(o_{h}\right)$, three different steps are followed: parent selection, crossover and mutation.

PARENT SELECTION: The first step is to select two parents, $P_{1}$ and $P_{2}$, used to create one offspring. In order to select $P_{1}$, a binary tournament selection is performed. Two individuals are selected randomly from the population and the best one will be selected as a parent. In order to decide which individual is better, first the front is compared, and the individual belonging to a better front is selected. If they belong to the same front, the individual with a higher CD is selected to promote diversity. The same procedure is followed to select $P_{2}$.

CROSSOVER: Once the two parents are selected $\left(P_{1}\right.$ and $\left.P_{2}\right)$, the $h$-th offspring $\left(o_{h}\right)$ is created by a two step crossover (due to the hierarchy). Firstly, the information of the $i$-th channel of the $o$-th offspring $\left(o_{h} \cdot x_{i}\right)$ is randomly taken with a probability $C R_{1} \in[0,1]$ from $P_{1}\left(P_{1} \cdot x_{i}\right)$, otherwise it is taken from $P_{2}\left(P_{2} \cdot x_{i}\right)$. Secondly, the information of the $j$-th frequency of the $i$-th channel for the $o$-th offspring $\left(o_{h} \cdot y_{i, j}\right)$ is randomly taken with a probability $C R_{2} \in[0,1]$ from $P_{1}\left(P_{1} \cdot y_{i, j}\right)$, otherwise it is taken from $P_{2}\left(P_{2} \cdot y_{i, j}\right)$.

MUTATION: The last step is to mutate the new offspring in order to diversify from the two parents and explore the search space. As in crossover, a two-step mutation has to be performed to deal with the hierarchical representation. The first step is to mutate the vector with all the information about the channels $\left(o_{h} \cdot x\right)$. Each value inside $o_{h} . x$ will have a probability of $M P_{1}$ of being changed from 0 to 1 , or vice versa. In the second step, the vectors holding the frequency information of all the channels $\left(o_{h} . y\right)$ are mutated. Similarly to the first step, all the values inside $o_{h} \cdot y$ will be mutated with a probability of $M P_{2}$.

\subsection{Objective functions}

There are two main objectives that will always be used in combination with others: Pearson correlation of the selected features (PCFS) and Feature reduction (FR). PCFS and FR are calculated as described in Eq. (1) and Eq. (2), respectively.

$$
\begin{gathered}
\text { PCFS }=\frac{\mid \text { featu } \mid \cdot \overline{\rho_{s, y}}}{\sqrt{\mid \text { featu }|+| \text { featu } \mid \cdot(\mid \text { featu } \mid-1) \cdot \overline{\rho_{s, r}}}} \\
F R=1-\frac{\mid \text { featu } \mid}{\mid \text { totalfeatu } \mid}
\end{gathered}
$$

where $\mid$ featu $\mid$ is equal to the number of features selected by NSGAII and $\mid$ totalfeatu $\mid$ is equal to the maximum number of features. Additionally, $\overline{\rho_{s, y}}$ is the average value of the Pearson's correlation coefficient ${ }^{1}$ between the selected features and the classes,

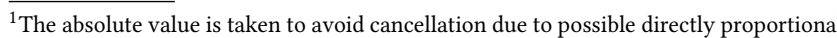
and inversely proportional relation between features or between features and classes.
}

while $\overline{\rho_{s, r}}$ represents the average value of the Pearson's correlation coefficient ${ }^{1}$ between all selected features.

A total of 7 combinations of objectives have been studied. In all the studied combinations, PCFS or/and FR are included as one of the objective. The objective combinations are: PCFS - FR, PCFS SVML, FR - SVML, PCFS - ANN, FR - ANN, PCFS - k-NN, and FR $\mathrm{k}-\mathrm{NN}$.

If a classifier is used as one of the objectives, the objective value is calculated as indicated in Eq. (3).

$$
\text { objectiveValue }=\frac{\frac{A c c u 1+A c c u 2}{2}+\operatorname{minAccu}}{2}
$$

where Accu1 and Accu2 stand for the accuracy obtained when predicting the first and the second class, respectively. minAccu represent the minimum value between $A c c u 1$ and Accu2. The reason of calculating the objective value this way is to avoid finding a solution in which the classifier predicts the same class all the time in imbalanced class distributions.

\section{EXPERIMENTATION}

This section describes the details of the experimentation and how it was implemented.

\subsection{MI data set description}

Data was collected from 6 healthy subjects (4 male, 2 females, mean \pm std age of $33.5 \pm 12.4$ ) while they were engaged in a task with interleaved Motor Imagery (MI) and Motor Execution (ME) trials. Although data during ME was discarded (out of the scope for this work), it is included in the task description for completeness. Seated comfortably in front of a computer screen, subjects were first instructed on which hand movement to execute (ME) or imagine (MI) within the block (either open or close their right hand). A block consisted of one ME trial followed by one MI trial. A visual (and auditory) instruction was presented for $4 \mathrm{~s}$ after which a focus point appeared for $2 \mathrm{~s}$, followed by a brief auditory tone that instructed the subjects to start performing the task (ME or MI of open or close hand). Subjects were given $4 \mathrm{~s}$ to perform each task with prior instructions to execute/imagine the movement only once with eyes open. At the end of each block, subjects were asked to rate their own performance of the MI. Further details on the task can be found in [39].

The data set in this study contains two different classes: imaginary movements of the right hand and rest recorded using 64 Active $\mathrm{Ag} / \mathrm{AgCl}$ electrodes (ActiChamp, Brain Products). The electrodes were positioned according to the international 10/20 system [10]. Two of the electrodes were located elsewhere (below right eye for EoG-artifacts and on right nose wing for reference). 42 channels were selected for analysis: AFz, Fz, F1-4, FCz, FC1-6, Cz, C1-6, CPz, CP1-6, Pz, P1-6, POz, PO3-4, PO7-8, Oz, O1-2, since the eccentric channels are prone to eye- and muscle artifacts (Figure 1). From each subject, a total of 240 samples were recorded (120 for each class) with a sampling frequency of $1000 \mathrm{~Hz}$ and band pass-filtered from 1-100 Hz. Surface Laplacian [4] was then applied to spatially filter the data. Spectral power of frequencies between 2 and $60 \mathrm{~Hz}$ was calculated for each channel using complex Morlet Wavelets [40]. This span of frequencies has previously been successfully 
used to classify imagined movements[31]. Power-data was downsampled to $40 \mathrm{~Hz}$ in order to reduce the size and make the data set more manageable. Then, for each subject, each channel and each frequency, power was normalized between 0 and 1 . Data was averaged across the time windows from 1000 to $25 \mathrm{~ms}$ before the auditory tone (start signal), assigned as baseline/rest, capturing the last part of the preparation period (focus point is displayed), and time windows from 475 to $1450 \mathrm{~ms}$ after the auditory tone, assigned as MI. Time windows from 0 to $450 \mathrm{~ms}$ were discarded to avoid overlap between the rest and the MI task signals. Further details on the data set and preprocessing can be found in [23].

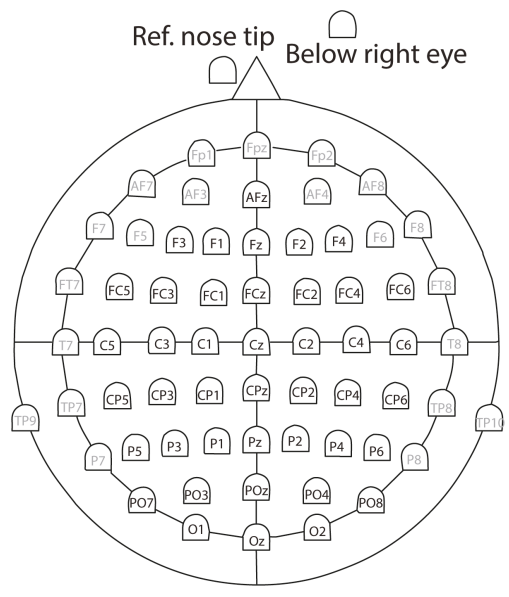

Figure 1: EEG electrode loyout. Electrodes included in data analysis appear in black text, discarded in grey text.

\subsection{Hierarchical NSGA-II}

The NSGA-II has been implemented by the authors in Matlab. The parameters of the algorithm are listed below:

- Population Size: 15

- Crossover Rate $1\left(C R_{1}\right): 0.5$

- Crossover Rate $2\left(C R_{2}\right): 0.5$

- Mutation Probability $1\left(M P_{1}\right): 0.05$

- Mutation Probability $2\left(M P_{2}\right): 0.01$

- Maximum generation $\left(g_{\max }\right): 3000$

\subsection{Tested classifiers}

Four different classifiers are compared in this study: linear discriminant analysis, support vector machine, artificial neural network and k-nearest neighbour. These classifiers have been implemented in Matlab.

Linear Discriminant Analysis (LDA): LDA has been frequently applied to EEG MI data sets $[1,30]$. As a binary classifier, it attempts to find a hyperplane $(w)$ that is able to separate between two classes [38]. The goal is to find the parameters, also called weights $(w)$, that defines the hyperplane, such that the mean square error of $y=w \cdot x$, where $y$ represents the class and $x$ the input data, is minimized. If the data is linearly separable, LDA will easily find a hyperplane separating the classes and will thus generate a high classification accuracy. The LDA algorithm has been implemented using the 'fircdiscr' function with default values and optimizing delta and gamma using Bayesian optimization.

Support Vector Machine (SVM): Similar to LDA, SVM also aims to find a hyperplane to separate data into the two classes [33, 38]. However, this hyperplane is positioned with the maximum margin to the support vectors (solutions near the hyperplane). Because of this, it often generalizes well since it maximizes the separation between the hyperplane and the classes. SVM carries the advantage over LDA that, by using a kernel function that projects the data into a higher dimensional space, it can separate data containing nonlinearities. SVMs have been widely used in many EEG applications with a high success $[16,22]$. Here, two SVM algorithms have been implemented using 'fitcsvm' function; a linear kernel (SVML) and a polynomial kernel (SVMP). All the other parameters were set to default values.

Artificial Neural Network (ANN): The ANN, inspired by the human brain, is built up by neurons and connections between them [18]. The basic principle of ANN is similar as in LDA and SVM: find the optimal hyperplane that separates two classes. Actually, a single layer ANN work similar to the LDA. The advantage of ANN is that more than one layer can be included into the model, enabling more complex separation boundaries between the classes without the requirement of linearity. However, ANN is prone to over-fitting if the dimensionality of the problem is high compared to the number of training samples. Despite of this, ANN has obtained good performance in many EEG applications [6, 12]. In our work, the Adam optimizer of the Matlab toolbox has been used as the optimization method, with an initial learning rate of 0.01 and the maximum number of epochs set to 300 . The structure of the network is described below:

- 1st Fully connected layer (hidden layer): number of neurons equal to 0.2 multiplied by the number of inputs with ReLU as the activation function.

- 2nd Fully connected layer (output layer): number of neurons equal to the number of classes (2) with softmax as the activation function.

- Classification layer.

$k$ - Nearest Neighbour $(k-N N)$ : The k-NN algorithm is one of the simpler supervised learning classification methods [7, 33]. When a new sample, $x$, is presented to the classifier, a metric, $d$, (e.g. the Euclidean distance) is calculated between $x$ and each training sample. The class of the $\mathrm{k}$ nearest neighbours are taken into consideration and a voting procedure is performed. The new sample will be classified as the class with the higher number of votes. In theory, with a sufficient high value of $\mathrm{k}$ and enough training samples, $\mathrm{k}-\mathrm{NN}$ could approximate any function. However, K-NN suffers when the dimensionality of the problem increases, specifically if the number of training samples is small (i.e. as in the used MI data set). k-NN has been used in many BCI applications [17, 20]. The k-NN used in this work calculates the Euclidean distance to be used as the distance measure the dissimilarity and $\mathrm{k}$ is set to 5 .

\subsection{Classification training/testing procedure}

Due to the small number of examples and the high number of features, we decided to apply a 10 -fold cross-validation for training/testing in order to avoid over-fitting. The NSGA-II has therefore 
been independently applied 10 times to each test subject. Additionally, if a classifier is used as one of the objectives, then, an extra 5 -folder has been applied during the training (the average accuracy, of the 5 different folders, is taken as the fitness value). Because our work focuses on a deepened investigation of the impact of different objectives, the reported classification accuracies of the NSGA-II, unless otherwise stated, represent the average classification accuracy across the individuals on the pareto-front.

\subsection{Statistical validation}

To statistically assess whether a set of paired samples come from the same distribution, the non-parametric Wilcoxon's signed rank test was used. As normality of the data could not be assumed, this statistical test was used for all comparisons, unless otherwise stated.

\section{RESULTS}

\subsection{Performance of different objectives in NSGA-II}

In this section, different classifiers (ANN, SVML, k-NN and LDA) are combined and used as objective functions either with PCFS or with FR. Additionally, PFCS is also combined with FR. The different objective pairs are evaluated with regards to classification accuracy, using five different classifiers (LDA, SVML, SVMP, ANN and k$\mathrm{NN}$ ) and feature reduction ratio. The results are shown in Table 1. Classification accuracies are averaged across subjects.

The objective-combinations, in which PFCS is involved, obtained better classification accuracy (average across all classifiers) as compared to the other objective-combinations (the statistical comparisons are given in Table 2). Having FR as an objective (instead of PCFS) degraded the classification accuracy with 3-6\% while decreasing the number of features approximately $3 \%$ more. With consideration to feature reduction, the objective combination that used FR as one of the objectives apply a reduction of the features of around $99,5 \%$.

When comparing different classifiers for testing, the LDA systematically obtained the best classification accuracy for all objectivecombinations (the statistical comparisons are given in Table 3). It is interesting to note that even though $\mathrm{k}-\mathrm{NN}$ as an objective in combination with PCFS yielded the highest averaged classification accuracy, the use of k-NN as the classifier decreased classification accuracy dramatically. An average of $10 \%$ degradation can be observed across all objective-combinations when k-NN was used as a classifier in testing and it was systematically outperformed by the other classifiers $(p<0.001)$. There is one exception. When the combination FR - k-NN is used, the accuracy of $\mathrm{k}-\mathrm{NN}$ is similar or better (up to 5,5\% increase) as compared to the other classifiers.

\subsection{Correlations between objectives and classifiers}

In this section, the correlation between the objectives and between the objectives and classifiers are studied in order to gain an understanding of whether certain dependencies are favorable for classification. Spearman's correlation coefficients are calculated and results can be found in Figure 2, where coefficients equal to 1 signify perfect positive correlation, coefficients equal to -1 signify perfect negative correlation and coefficients equal to 0 signify no correlation at all.

First, it can be noted that PCFS has a significant positive correlation with the ojectives using a classification algorithm (SVML, ANN, k-NN and LDA; $\mathrm{p}<0.05$ ), having strongest correlation with SVML ( $\mathrm{r}=0.57)$ and weakest correlation with LDA $(\mathrm{r}=0.28)$. Comparing these correlation coefficients to the classification accuracy in table 1 shows that there is no apparent relationship between these measures.

However, when paired with FR, significant negative correlations can be observed for objective-combinations, PCFS - FR, FR - SVML and FR - k-NN ( $\mathrm{p}<0.05)$, pointing to the competitive relation between these objectives. On the other hand, no correlation was found between objective combinations, FR - LDA and FR-ANN ( $>>0.05)$. Comparing to the classification accuracy suggests that a significant trade-off relationship between FR and an objective classifier yields better classification accuracy.

With regards to how the different objectives relate to the classifier during testing, a strong significant correlation between both objectives and all the classifiers $(\mathrm{p}<0.05)$ is observed when PCFS is used together with a classifier as a objective pair. On the other hand, if FR is used as one of the objectives, two different outcomes are possible. Firstly, if a negative correlation between FR and the other objective exist (PCFS - FR, FR - SVML and FR - LDA), then FR has a small negative correlation with all the classifiers. Secondly, if no correlation exist between FR and the other objective (FR - ANN and FR - LDA), then FR has a small positive correlation with all the classifiers. It can be further noted that if a specific classification algorithm is used as one of the objectives, it does not necessarily translate into a stronger correlation between the same algorithm as a classifier. One exception to this was the k-NN classifier, which was more strongly correlated to the k-NN objective and substantially less correlated to the other classification objective algorithms.

This, in accordance with results in the previous paragraph, suggests that there is a similarity between all of the classifiers except for the k-NN.

\subsection{Impact of NSGA-II objectives on feature selection}

Medical applications of MI feature selection and classification are often targeted towards rehabilitation aiming to improve motor recovery of people who has suffered from brain injury. Within a closed-loop neurofeedback system, the rehabilitee is instructed to modulate EEG features functionally relevant to MI and thereby enhance motor-related activity and promote functional reorganization of motor-related networks [36]. From this, it is evident that the feature selection is crucial in producing meaningful rehabilitation.

In this section, we investigate the similarity of feature selection using different objectives in NSGA-II. For this purpose, the cosine similarity was calculated between the selected features of all pairwise objective-combinations as it allows to measure similarities of selected features. The results are shown in Table 4, where values close to 1 signifies high similarity in selected features and values close to 0 indicates that the algorithms selected different features. 
Table 1: Comparison of the classification accuracy and the percentage of feature reduction obtained by NSGA-II, using different pairs of training objectives and different classifiers.

\begin{tabular}{|ll||c|c|c|c|c|c||c|}
\hline \multirow{2}{*}{ OBJECTIVE 1 } & \multirow{2}{*}{ OBJECTIVE 2 } & \multicolumn{5}{c||}{ Classification Accuracy } & \multirow{2}{*}{ Feature reduction } \\
\cline { 3 - 8 } & FR & $74,22 \%$ & $74,28 \%$ & $69,77 \%$ & $72,24 \%$ & $59,68 \%$ & $70,04 \%$ & $99,50 \%$ \\
\hline \hline PCFS & SVML & $76,32 \%$ & $74,72 \%$ & $72,55 \%$ & $75,36 \%$ & $58,62 \%$ & $71,51 \%$ & $95,74 \%$ \\
\hline PCFS & ANN & $75,96 \%$ & $74,34 \%$ & $72,77 \%$ & $74,50 \%$ & $60,19 \%$ & $71,55 \%$ & $96,91 \%$ \\
\hline PCFS & k-NN & $75,90 \%$ & $74,50 \%$ & $71,29 \%$ & $74,91 \%$ & $69,66 \%$ & $\mathbf{7 3 , 2 5 \%}$ & $96,88 \%$ \\
\hline PCFS & LDA & $75,93 \%$ & $74,47 \%$ & $71,75 \%$ & $75,29 \%$ & $59,97 \%$ & $71,48 \%$ & $96,96 \%$ \\
\hline PCFS & SVML & $72,51 \%$ & $72,48 \%$ & $68,80 \%$ & $68,84 \%$ & $58,21 \%$ & $68,17 \%$ & $98,73 \%$ \\
\hline FR & ANN & $69,26 \%$ & $69,39 \%$ & $66,31 \%$ & $64,89 \%$ & $57,52 \%$ & $65,47 \%$ & $99,56 \%$ \\
\hline FR & k-NN & $71,92 \%$ & $71,73 \%$ & $67,85 \%$ & $64,57 \%$ & $70,10 \%$ & $69,23 \%$ & $99,50 \%$ \\
\hline FR & LDA & $69,14 \%$ & $69,24 \%$ & $66,13 \%$ & $64,20 \%$ & $59,11 \%$ & $65,57 \%$ & $\mathbf{9 9 , 6 4 \%}$ \\
\hline FR & Average & $\mathbf{7 3 , 4 6 \%}$ & $72,80 \%$ & $69,69 \%$ & $70,53 \%$ & $61,45 \%$ & - & $98,16 \%$ \\
\hline \hline \multicolumn{2}{|c|}{} & & & & & & & \\
\hline
\end{tabular}

Table 2: Wilcoxon's statistical test $(\alpha=0.05)$ between the different pairs of objectives (left vs top). Classification accuracies above the 95\%-confidence interval are denoted with ${ }^{, *}, p<0.05,{ }^{* * *} p<0.01,{ }^{* * *}, p<0.001$.

\begin{tabular}{|c|c|c|c|c|c|c|c|c|c|c|c|c|}
\hline & Obj 1 Obj 2 & Obj 1 Obj 2 & Obj 1 Obj 2 & Obj 1 Obj 2 & Obj 1 Obj 2 & $\mathrm{Obj}$ & $1 \mathrm{Obj} 2$ & Obj & 1 Obj 2 & $\mathrm{Ob}$ & $1 \mathrm{Obj} 2$ & Obj 1 Obj 2 \\
\hline Obj 1 Obj 2 & PCFS FR & PCFS SVML & PCFS ANN & PCFS k-NN & PCFS LDA & FR & SVML & FR & ANN & FR & k-NN & $\begin{array}{ll}\text { FR } & \text { LDA } \\
\end{array}$ \\
\hline PCFS FR & - & & & & & & $z_{* * *}$ & & *** & & & $2 * * *$ \\
\hline PCFS SVML & ** & - & & & & & $* * *$ & & *** & & ** & $* * *$ \\
\hline PCFS ANN & ** & & - & & & & $* * *$ & & $* * *$ & & $* *$ & $* * *$ \\
\hline PCFS k-NN & $* * *$ & & & - & & & $* * *$ & & $* * *$ & & $* * *$ & *** \\
\hline PCFS LDA & ** & & & & - & & $* * *$ & & $* * *$ & & ** & $* * *$ \\
\hline $\begin{array}{ll}\text { FR } & \text { SVML } \\
\end{array}$ & & & & & & & - & & *** & & & $* * *$ \\
\hline $\begin{array}{ll}\text { FR } & \text { ANN } \\
\end{array}$ & & & & & & & & & - & & & \\
\hline $\begin{array}{ll}\text { FR } & \text { k-NN } \\
\end{array}$ & & & & & & & & & ** & & - & ** \\
\hline FR $\quad$ LDA & & & & & & & & & & & & - \\
\hline
\end{tabular}

Table 3: Wilcoxon's statistical test $(\alpha=0.05)$ between different classifiers (left vs top). Classification accuracies above the 95\%-confidence interval are denoted with ${ }^{*}{ }^{*} \mathrm{p}<0.05$, ${ }^{* *}$, $\mathbf{p}<0.01,{ }^{* * *}, \mathbf{p}<0.001$.

\begin{tabular}{|l|c|c|c|c|c|}
\hline & LDA & SVML & SVMP & ANN & k-NN \\
\hline LDA & - & ${ }^{* * *}$ & ${ }^{* *}$ & ${ }^{* * *}$ & ${ }^{* * *}$ \\
\hline SVML & & - & ${ }^{* * *}$ & ${ }^{* * *}$ & ${ }^{* * *}$ \\
\hline SVMP & & & - & & ${ }^{* * *}$ \\
\hline ANN & & & ${ }^{* *}$ & - & ${ }^{* * *}$ \\
\hline k-NN & & & & & - \\
\hline
\end{tabular}

It can be observed that all classifier objectives combined with PCFS had high cosine similarity $(>0.75)$ indicating that although different objective-combinations, these algorithms selected very similar features related to MI.

In order to take a closer look at the selected features, the PCFS combined with k-NN (which produced the highest classification accuracy) and FR (which generated one of the highest feature reduction), respectively, are plotted as topographical maps to show channels with a high ranking score (i.e. percentage of which each feature was selected in the pareto front individuals, Figure 3), and as graphs to show the high-ranked frequencies within the high-ranked channels (thick-dotted channels, all subjects: rank $>0.4$ and subject M: rank $>0.6$ ) (Figure 3). When investigating feature rank across all subjects, these objective-combinations predominantly selected electrodes contralaterally located over the sensorimotor area (i.e. channels C3, CP3) (figure 3b.1 and 3b.2). Despite selecting similar channels, the frequency rank was rather different with a narrow high-rank frequency band centered at $9.2 \mathrm{~Hz}$ for objectives FR and $\mathrm{k}-\mathrm{NN}$ as compared to objectives PCFS and k-NN in which a broader high-rank frequency band ranging from $8 \mathrm{~Hz}$ to $20 \mathrm{~Hz}$ was present together with higher frequencies around $35 \mathrm{~Hz}$. As a comparison, objectives PCFS and FR selects similar channels as the other two objective pairs although with slightly more emphasis on the ipsilateral side. Regarding frequencies, PCFS-FR objectives have a slightly broader high-rank frequency band centered at $9.2 \mathrm{~Hz}$ (ranging between $8.2 \mathrm{~Hz}$ to $13.1 \mathrm{~Hz}$ ) but higher frequencies have low rank. It is clear that although dissimilarities exist in frequency rank, all three objective pairs rank $9.2 \mathrm{~Hz}$ frequency as highest. At a subject-level, differences of feature ranking between objective pairs increase (subject $M$ is plotted as a representative example). Activation patterns in EEG frequency rhythms related to MI have been well described in the literature[28]. Based on these previous findings, we would expect to select channels over the sensorimotor cortex contralateral (around C3 and CP3), but possibly also ipsilateral (around C4 and 


\begin{tabular}{|c|c|c|c|c|c|c|c|c|c|}
\hline \multicolumn{2}{|c|}{ Training Objectives } & \multicolumn{3}{|c|}{ Training algorithms/metrics } & \multicolumn{5}{|c|}{ Test Algorithms } \\
\hline Objective 1 & Objective 2 & & PCFS & $\mathrm{FR}$ & LDA & SVML & SVMP & ANN & k-NN \\
\hline \multirow{2}{*}{ PCFS } & \multirow{2}{*}{ FR } & PCFS & - & $-0,371$ & 0,665 & 0,640 & 0,572 & 0,578 & 0,408 \\
\hline & & $\mathrm{FR}$ & $-0,371$ & - & $-0,185$ & $-0,162$ & 0,027 & $-0,263$ & 0,032 \\
\hline Objective 1 & Objective 2 & & PCFS & SVML & LDA & SVML & SVMP & ANN & k-NN \\
\hline \multirow{2}{*}{ PCFS } & \multirow{2}{*}{ SVML } & PCFS & - & 0,586 & 0,660 & 0,615 & 0,687 & 0,637 & 0,322 \\
\hline & & SVML & 0,586 & - & 0,555 & 0,512 & 0,583 & 0,555 & 0,369 \\
\hline Objective 1 & Objective 2 & & PCFS & ANN & LDA & SVML & SVMP & ANN & k-NN \\
\hline \multirow{2}{*}{ PCFS } & \multirow{2}{*}{ ANN } & PCFS & - & 0,428 & 0,703 & 0,656 & 0,705 & 0,650 & 0,369 \\
\hline & & ANN & 0,428 & - & 0,441 & 0,368 & 0,380 & 0,390 & 0,223 \\
\hline Objective 1 & Objective 2 & & PCFS & k-NN & LDA & SVML & SVMP & ANN & k-NN \\
\hline \multirow{2}{*}{ PCFS } & \multirow{2}{*}{ k-NN } & PCFS & - & 0,295 & 0,631 & 0,601 & 0,649 & 0,608 & 0,554 \\
\hline & & k-NN & 0,295 & - & 0,393 & 0,398 & 0,402 & 0,400 & 0,641 \\
\hline Objective 1 & Objective 2 & & PCFS & LDA & LDA & SVML & SVMP & ANN & k-NN \\
\hline \multirow{2}{*}{ PCFS } & \multirow{2}{*}{ LDA } & PCFS & - & 0,280 & 0,702 & 0,669 & 0,635 & 0,684 & 0,206 \\
\hline & & LDA & 0,280 & - & 0,340 & 0,272 & 0,309 & 0,306 & 0,133 \\
\hline Objective 1 & Objective 2 & & $\mathrm{FR}$ & SVML & LDA & SVML & SVMP & ANN & k-NN \\
\hline \multirow{2}{*}{ FR } & \multirow{2}{*}{ SVML } & FR & - & $-0,491$ & $-0,173$ & $-0,126$ & $-0,109$ & $-0,258$ & $-0,010$ \\
\hline & & SVML & $-0,491$ & - & 0,644 & 0,633 & 0,577 & 0,606 & 0,306 \\
\hline Objective 1 & Objective 2 & & $F R$ & ANN & LDA & SVML & SVMP & ANN & k-NN \\
\hline \multirow{2}{*}{ FR } & \multirow{2}{*}{ ANN } & $\mathrm{FR}$ & - & 0,026 & 0,164 & 0,197 & 0,230 & 0,091 & 0,144 \\
\hline & & ANN & 0,026 & - & 0,474 & 0,473 & 0,491 & 0,381 & 0,284 \\
\hline Objective 1 & Objective 2 & & $\mathrm{FR}$ & k-NN & LDA & SVML & SVMP & ANN & k-NN \\
\hline \multirow{2}{*}{ FR } & \multirow{2}{*}{ k-NN } & $\mathrm{FR}$ & - & $-0,408$ & $-0,248$ & $-0,213$ & $-0,106$ & $-0,385$ & $-0,254$ \\
\hline & & k-NN & $-0,408$ & - & 0,714 & 0,707 & 0,629 & 0,517 & 0,670 \\
\hline Objective 1 & Objective 2 & & FR & LDA & LDA & SVML & SVMP & ANN & k-NN \\
\hline \multirow{2}{*}{$F R$} & \multirow{2}{*}{ LDA } & $\mathrm{FR}$ & - & $-0,083$ & 0,070 & 0,157 & 0,149 & 0,010 & 0,136 \\
\hline & & LDA & $-0,083$ & - & 0,433 & 0,461 & 0,415 & 0,361 & 0,268 \\
\hline
\end{tabular}

Figure 2: Spearman's correlation coefficients between different objectives and between objectives and classifiers. Values in boldface signify statistically significant correlations with $95 \%$-confidence. Positive correlations are represented with a green color, while negative correlations are represented with a red color. More intensity indicates a stronger correlation.

$\mathrm{CP} 4)$ to the imagined hand, and select frequencies around $8-12 \mathrm{~Hz}$ (alpha) and $14-25 \mathrm{~Hz}$ (beta). Judging from these expectations, the selected features using objectives PCFS-classifier (PCFS-k-NN shown in figure 2 as a representative example of objectives PCFS-classifier) across all subjects seem to select all of the previously described features related to MI, while the other objective-pairs selected only a subset.

Depending on the subject, we encountered some variability in the highly ranked features, with some subjects displaying small displacements in highly ranked channels and peak frequencies. This is in accordance to previous literature reporting individual variability during MI [28, 34]. Figures 3a.1 and 3a.2 shows one subject, from which has visibly different high-ranked features as compared to the average across all subjects.

Furthermore, objective-combinations with FR (exception of PCFSFR) had lower similarity to all other objectives $(<0.56)$ indicating that NSGA-II with these objectives selected different features. Topographical brain maps and frequency amplitude graphs support this indication as we observed partly different high-rank features. The objective-combination FR-k-NN is plotted in Figures 3a.3 to show an example but similar differences hold for the other objectivecombinations with FR.

\section{CONCLUSION}

In this work, a multi-objective GA, NSGA-II, is used in combination with a hierarchical individual representation in order to select features functionally relevant to MI. Different objectives and classifiers were evaluated with regards to classification accuracy, feature reduction and selected features. The work has four main outcomes. 1) The LDA classifier obtains the best classification accuracy independently of the objectives, 2) having FR as an objective is not necessary for an effective feature reduction as objectives PCFSclassifier achieves up to almost $97 \%$ feature reduction, 3) FR as an objective increases the variability of the selected features, and 4) PCFS-classifier as objectives generates highly similar feature rank.

In conclusion, considering feature selection, reduction of features and classification accuracy, the NSGA-II using the objectives PCFS and a classifier, is a good method as it produces a trade-off solution with relatively high accuracy while reliably selecting a small number of features functionally relevant to MI.

\section{REFERENCES}

[1] J. Asensio-Cubero, J.Q. Ga, and R. Palaniappan. 2013. Multiresolution analysis over simple graphs for brain computer interfaces. Fournal of Neural Engineering 10, 4 (2013), 046014

[2] T Back and H P Schwefel. 1993. An overview of evolutionary algorithms for parameter optimization. Evolutionary computation 1, 1 (1993), 1-23. 
Table 4: Cosine similarities of selected features between different pairs of objectives. Values in boldface point out similarities above 0.75 .

\begin{tabular}{|c|c|c|c|c|c|c|c|c|c|}
\hline & Obj 1 Obj 2 & Obj 1 Obj 2 & Obj 1 Obj 2 & Obj 1 Obj 2 & Obj 1 Obj 2 & Obj 1 Obj 2 & Obj 1 Obj 2 & Obj 1 Obj 2 & Obj 1 Obj 2 \\
\hline Obj 1 Obj 2 & $\begin{array}{ll}\text { PCFS } & \text { FR }\end{array}$ & PCFS SVML & PCFS ANN & PCFS k-NN & PCFS LDA & FR $\quad$ SVML & FR $\quad$ ANN & $\begin{array}{ll}\text { FR } & k-N N \\
\end{array}$ & FR LDA \\
\hline PCFS FR & - & 0,58 & 0,64 & 0,58 & 0,61 & 0,52 & 0,35 & 0,41 & 0,42 \\
\hline PCFS SVML & & - & 0,85 & $\mathbf{0 , 7 7}$ & 0,83 & 0,55 & 0,43 & 0,40 & 0,46 \\
\hline PCFS ANN & & & - & 0,78 & 0,86 & 0,52 & 0,40 & 0,40 & 0,44 \\
\hline PCFS k-NN & & & & - & 0,79 & 0,48 & 0,39 & 0,55 & 0,41 \\
\hline PCFS LDA & & & & & - & 0,52 & 0,41 & 0,42 & 0,43 \\
\hline FR $\quad$ SVML & & & & & & - & 0,38 & 0,38 & 0,38 \\
\hline ANN & & & & & & & - & 0,27 & 0,33 \\
\hline k-NN & & & & & & & & - & 0,28 \\
\hline LDA & & & & & & & & & - \\
\hline
\end{tabular}

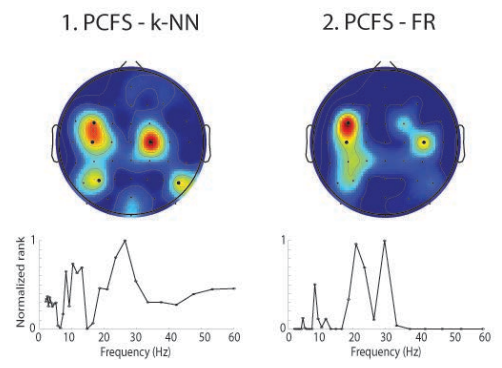

(a) Subject D
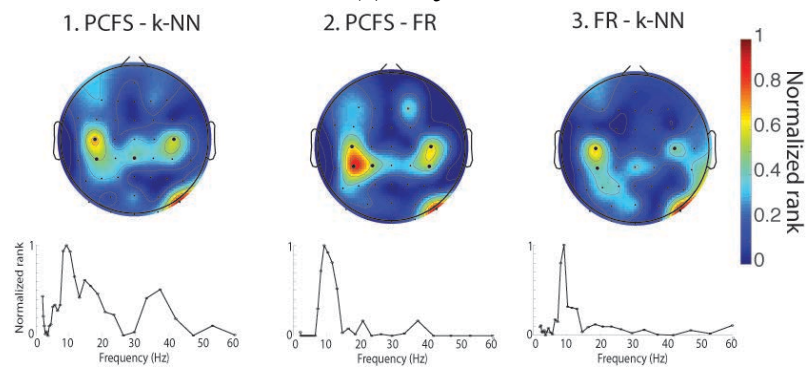

(b) All subjects

Figure 3: NSGA-II high-ranked features using different objectives. Topographical brain maps depict normalized average rank of channels, across all frequencies, for one subject (A) and all subjects (B) using different objectives. The normalized average rank of frequencies across thick-dotted channels (black: contralateral, grey: ipsilateral) are shown below each topography.

[3] C. Blum and A. Roli. 2003. Metaheuristics in combinatorial optimization: Overview and conceptual comparison. ACM computing surveys (CSUR) 35, 3 (2003), 268-308.

[4] Claudio Carvalhaes and J Acacio de Barros. 2015. The surface Laplacian technique in EEG: Theory and methods. International fournal of Psychophysiology 97, 3 (2015), 174-188.

[5] A. Chakraborty and A.K. Kar. 2017. Swarm intelligence: A review of algorithms In Nature-Inspired Computing and Optimization. Springer, 475-494.

[6] R. Chatterjee and T. Bandyopadhyay. 2016. EEG based Motor Imagery Classification using SVM and MLP. In 2nd International Conference on Computational Intelligence and Networks (CINE). 84-89.

[7] T.M. Cover and P. Hart. 1967. Nearest neighbor pattern classification. IEEE transactions on information theory 13, 1 (1967), 21-27.

[8] K Deb, S. Agrawal, A Pratap, and T. Meyarivan. 2000. A fast elitist non-dominated sorting genetic algorithm for multi-objective optimization: NSGA-II. In International conference on parallel problem solving from nature. Springer, Berlin, 849-858.
[9] K. Deb, A. Pratap, S. Agarwal, and T. MEyarivan. 2002. A fast and elitist multiobjective genetic algorithm: NSGA-II. IEEE Transaction on Evolutionary Computation 6, 2 (2002), 182-197.

[10] J.S. Ebersole and T.A. Pedley. 2003. Current practice of clinical electroencephalography. Lippincott Williams \& Wilkins.

[11] H.H. Ehrsson, S. Geyer, and E. Naito. 2003. Imagery of voluntary movement of fingers, toes, and tongue activates corresponding body-part-specific motor representations. F. Neurophysiol. 90 (2003), 3304-3316.

[12] L. Fabien, L. Anatole, L. Fabrice, and A. Bruno. 2007. Studying the use of fuzzy inference systems for motor imagery classification. IEEE transactions on neural systems and rehabilitation engineering 15, 2 (2007), 322-324.

[13] D E Goldberg. 1989. Genetic Algorithm in Search, Optimization and Machine Learning. In Addison-Wesley, New York.

[14] M. Gong and Y.H. Yang. 2001. Multi-resolution stereo matching using genetic algorithm. In IEEE workshop on Stereo and Multi-Baseline Vision. 21-29.

[15] Jesús González, Julio Ortega, Miguel Damas, Pedro Martín-Smith, and John Q. Gan. 2019. A new multi-objective wrapper method for feature selection - Accuracy and stability analysis for BCI. Neurocomputing 333 (2019), 407-418. https: //doi.org/10.1016/j.neucom.2019.01.017

[16] E. Gysels, P. Renevery, and P. Celka. 2005. SVM-based recursive feature elimination to compare phase synchronization computed from broadband and narrowband EEG signals in brain-computer interfaces. Signal Processing 85, 11 (2005), $2178-2189$.

[17] I.T. Hettiarachchi, T.T. Nguyen, and S. Nahavandi. 2014. Motor imagery data classification for BCI application using wavelet packet feature extraction. In International Conference on Neural Information Processing. 519-526.

[18] A.K. Jain, J. Mao, and K.M. Mohiuddin. 1996. Artificial neural networks: A tutorial. Computer 3 (1996), 31-44.

[19] Chea Yau Kee, S. G. Ponnambalam, and Chu Kiong Loo. 2015. Multi-objective genetic algorithm as channel selection method for P300 and motor imagery data set. Neurocomputing 161, Mi (2015), 120-131. https://doi.org/10.1016/j.neucom. 2015.02.057

[20] R Khosrowabadi, R. Ouek, C. Ang, K.K. Tung, and M. Heijnen. 2011. A BrainComputer Interface for classifying EEG correlates of chronic mental stress. In International Joint Conference on Neural Networks. 757-762.

[21] S Kirkpatrick, C D Gelantt, and M P Vecchi. 1983. Optimization by simulated annealing. Science 220 (1983), 671-680.

[22] H. Lee and S. Choi. 2003. Pca+hmm+svm for eeg pattern classification. In Seventh International Symposium on Signal Processing and Its Applications. 541-554.

[23] Miguel Leon, Joaquin Ballesteros, Jonatan Tidare, Ning Xiong, and Elaine Astrand. 2019. Feature Selection of EEG OScillatory Activity Related to Motor Imagery Using a Hierarchical Genetic Algorithm. IEEE Congress on Evolutionary Computation (2019), 8.

[24] J. Lv and M. Liu. 2008. Common Spatial Pattern and Particle Swarm Optimization for Channel Selection in BCI. In 3rd International Conference on Innovative Computing Information and Control. 457-457.

[25] A Madureira, C Ramos, and S do Carmo Silva. 2002. A Coordination MEchanism for Real World Scheduling Problems using Genetic Algorithms. In IEEE Congress on Evolutionary Computation. 175-180.

[26] A. Mukhopadhyay, U. Maulik, S. Bandyopadhyay, and C. A. C. Coello. 2014. A Survey of Multiobjective Evolutionary Algorithms for Data Mining: Part I. IEEE Trans. Evol. Comput. 18 (2014), 4-19.

[27] G. Pfurtscheller and F.H. Lopes da Silva. 1999. Event-related EEG/MEG synchronization and desynchronization: basic principles. Clin. Neurophysiol. 110 (1999), 1842-1857.

[28] G. Pfurtscheller and C Neuper. 1997. Motor imagery activates primary sensorimotor area in humans. Neurosci. Lett. 239 (1997), 65-68.

[29] F Pichiorri, G. Morone, M. Petti, J. Toppi, I. Pisotta, M. Molinari, S. Paolucci, M. Inghilleri, L. Astolfi, F. Cincotti, and Et Al. 2015. Brain-computer interface boosts motor imagery practice during stroke recovery. Ann. Neurol. 77 (2015), 851-865. 
[30] A.C. Ramos, R.G. Hernandez, and M. Vellasco. 2016. Feature Selection methods applied to Motor Imagery task classification. In IEEE Latin American Conference on Computational Intelligence (LA-CCI). 1-6.

[31] Rinku Roy, Debdeep Sikdar, Manjunatha Mahadevappa, and CS Kumar. 2017. EEG based motor imagery study of time domain features for classification of power and precision hand grasps. In Neural Engineering (NER), 2017 8th International IEEE/EMBS Conference on. IEEE, 440-443.

[32] R Ruiz and T Stutzle. 2007. A simple and effective iterated greedy algorithm for the permutation flowshop scheduling problem. European fournal of Operational Research 177, 3 (2007), 2033-2049.

[33] S Russell and P Norvig. 2016. Artificial Intelligence: A Modern Approach. In Malaysia: Pearson Education Limited.

[34] A Schloegl, C Neuper, and G Pfurtscheller. 1997. Subject specific EEG patterns during motor imaginary [sic.: for imaginary read imagery]. In Proceedings of the 19th Annual International Conference of the IEEE Engineering in Medicine and Biology Society. Magnificent Milestones and Emerging Opportunities in Medical Engineering? (Cat. No.97CH36136, Vol. 4. 1530-1532.
[35] M. Schroder, M. Bogdan, T. Hinterberger, and N. Birbaumer. 2003. Automated EEG feature selection for brain computer interfaces. In First International IEEE EMBS Conference on Neural Engineering. 626-629.

[36] R Sitaram, T. Ros, L. Stoeckel, S. Haller, F. Scharnowski, J. Lewis-Peacock, N. Weiskopf, M.L. Blefari, M. Rana, E. Oblak, and Et Al. 2017. Closed-loop brain training: the science of neurofeedback. Nat. Rev. Neurosci. 18, 2 (2017), 86-100.

[37] N Srinivas and K. Deb. 1994. Muiltiobjective Optimization Using Nondominated Sorting in Genetic Algorithms. Evol. Comput. 2 (1994), 221-248.

[38] A. Subasi and M.I. Gursoy. 2010. EEG signal classification using PCA, ICA, LDA and support vector machines. Expert systems with applications 37, 12 (2010), 8659-8666.

[39] Jonatan Tidare, Miguel Leon, Ning Xiong, and E Astrand. 2019. Discriminating EEG spectral power related to mental imagery of closing and opening of hand. In 2019 9th International IEEE/EMBS Conference on Neural Engineering (NER). IEEE, 307-310.

[40] Christopher Torrence and Gilbert P Compo. 1998. A practical guide to wavelet analysis. Bulletin of the American Meteorological society 79, 1 (1998), 61-78. 